Journal of Fundamental and Applied Sciences

ISSN 1112-9867

Available online at

http://www.jfas.info

\title{
STUDIES OF CHANGES OF SOME BLOOD PARAMETERS AMONG WORKERS OF CEMENT OF EL MA EL ABIOD TEBESSA ALGERIA
}

\author{
M. Aouimeur ${ }^{1 *}$, T. Goudjil ${ }^{1}$, A. Rouabhia ${ }^{2}$, M. Hamiri $^{1}$ \\ ${ }^{1}$ Département de biologie, Université de Tébessa, Algérie. \\ ${ }^{2}$ Departement d'hydrogéologie, Université de Tébessa, Algérie.
}

Received: 13 July 2009 / Accepted: 09 December 2009 / Published online: 31 December 2009

\begin{abstract}
In Algeria, the industrial development engenders the appearance of the sanitary and environmental problems resulting from the pollution due to these certain industries such as the industry cimentière. With the aim of estimating the sanitary state of the workers of the cement works; a study was made for 30 workers of the cement works. This study is based on the determination of the values of 07 parameters hématologiques (red blood cells, haemoglobin, hématocrite, average spherical volume, The corpuscular concentration averages in haemoglobin, The corpuscular content averages in haemoglobin and the number of white blood cells) and two parameters considered as markers premature of the inflammation (sedimentation speed and protein-C-reactive). These results are compared with two other populations, one of the inhabitants of the region and other one of the inhabitants far from the cement works avec $60 \mathrm{~km}$. The statistical analyses are based on the comparison of the variances and the analysis in main constituent (ACP). The comparison of the variance of the various variable of the individuals show a resemblance between the workers and the inhabitants close to the cement works but significant differences appeared between both populations and those of the cement works for some parameters. The global analysis of these results with the ACP showed a general coherence. The observation of the groups allowed removing some peculiarities.
\end{abstract}

Author Correspondence, e-mail: meriemaoui@yahoo.fr

ICID: 1040457 
This distribution shows the relation between the functional state and the variation of the parameters.

Key words: industry cimentière, workers, signs of inflammation, comparison of the variance, analyzes in main constituent.

\section{INTRODUCTION}

La pollution est la présence dans l'atmosphère de substances étrangères à la composition normale de cette atmosphère et à des concentrations suffisamment élevées pour entrainer un impact sur l'homme, les animaux, les végétaux, les matériaux ou l'environnement d'une manière générale[5,9]. L'augmentation de la pollution atmosphérique pose de plus en plus des problèmes de santé publique cette dernière décennie, de nombreux travaux épidémiologiques ont montré que les niveaux modérés ou même faible de la pollution atmosphérique avait un impact à court terme sur la santé [7].

Le développement industriel engendre l'apparition des problèmes sanitaire et environnemental résultant de la pollution due à ces équipements est essentiellement l'industrie cimentière. Cette dernière est apparue remarquable sur le plan économique, social et ainsi environnemental ; dus à leur activité importante par la production du ciment ; produit à utilisation mondial très vaste, d'une part et d'autre part, par ces rejets atmosphériques qui ont une grande influence sur l'agriculture, la qualité des eaux, la santé humaine et même sur le climat de la région.

L'étude des effets des poussières sur l'homme est problème très vaste, très complexe et relativement peu exploré. Ceci en raison de variétés innombrables de polluants pouvant exister dans l'atmosphère d'origine naturelle ou artificielle.

Les principaux modes d'actions des poussières sur l'organisme résultent lorsqu'elles ne sont pas normalement éliminés ;

$\checkmark$ Soit de leur accumulation dans le système lymphatique pulmonaire.

$\checkmark$ Soit de leur transfert par le sang vers d'autres organes où elles sont susceptibles d'atteindre des concentrations toxiques, cet effet est lié essentiellement à l'absorption par les poumons, mais peut aussi intervenir par ingestion d'aliments contaminées, ou déglutition de poussières condensées dans le nez et la gorge. 
Plus les particules sont fines plus elles pénètrent profondément dans l'appareil respiratoire. Elles irritent le système respiratoire humain et peuvent contribuer au déclenchement des maladies respiratoires aigues [4,12].

La population la plus infectées par les activités d'une cimenterie est les travailleurs en premier lieu puis les habitants proches de la cimenterie. Ces gens sont en contacts directe à tout type de dégagements des cimenteries ce qui influe sur les différentes activités biologiques de leurs corps.

Dans le but d'évaluer l'état sanitaire des travaileurs de la cimenterie d' d'El Ma El Abiod et les habitants de la région environnante de la cimenterie, une étude a été faites pour déterminer les valeurs de 9 paramètres hématologiques et évaluer leurs varaitions par rapport à une population témoins dans la période de Avril à Septembre 2007

\section{MATÉRIELS ET MÉTHODES}

\section{Description du cite de travail}

\section{1. La plaine d'El Ma El Abiod}

El Ma El Abiod est une plaine à vocation agricole, de $420 \mathrm{Km}^{2}$ située au Sud Est de la ville de Tebessa, au Nord Est de l'Algérie. Elle est située à $26 \mathrm{Km}$ de la mer Méditerranée, à la limite de la frontière Algéro-tunisienne. La limite Nord de la plaine est formée par Dj Doukane, Dj Anoual et Dj Bouroumane.La limite Est de la plaine s'approche du territoire tunisien dans la région de Kodiat sidi Salah. A l'Ouest se trouve la plaine de Cheria. La limite Sud constituée par le synclinal de Bir Sbeikia.

L'économie de la région est basée sur l'agriculture et l'industrie minière. Elle est en effet à forte vocation pastorale et à d'ailleurs constitué une zone de parcours des troupeaux ovins jusqu'au début des années 1990. Depuis cette époque, la région connaît une activité industrielle très importante et consommatrice d'eau, représente principalement par l'industrie du ciment portland (ERCE), celle du verre (SOVEST) et celles des tubes roulés (ANABIB) [1].

\section{2. La Cimenterie ERCE}

La société a été crée au mois de Novembre 1993. Le siège social se trouve à Tébessa à $680 \mathrm{Km}$ au Sud Est d'Alger et à $250 \mathrm{Km}$ de la ville d'Annaba au Nord. La cimenterie se trouve à $26 \mathrm{Km}$ au Sud de Tébessa et à $35 \mathrm{Km}$ des frontières Algéro tunisienne, elle est la dernière réalisation publique dans le secteur du ciment. La cimenterie à une 
capacité de1600 tonnes/jours de clinker correspond à 500000 tonnes/an. Elle est dotée d'une ligne de la cuisson par voie sèche. Elle puise ses principales matières premières à partir des gisements proche du cite (calcaire : 500m et argile : 10Km). Les réserves de matières premières permettent une exploitation de plus de 100 ans. (Figure 1)

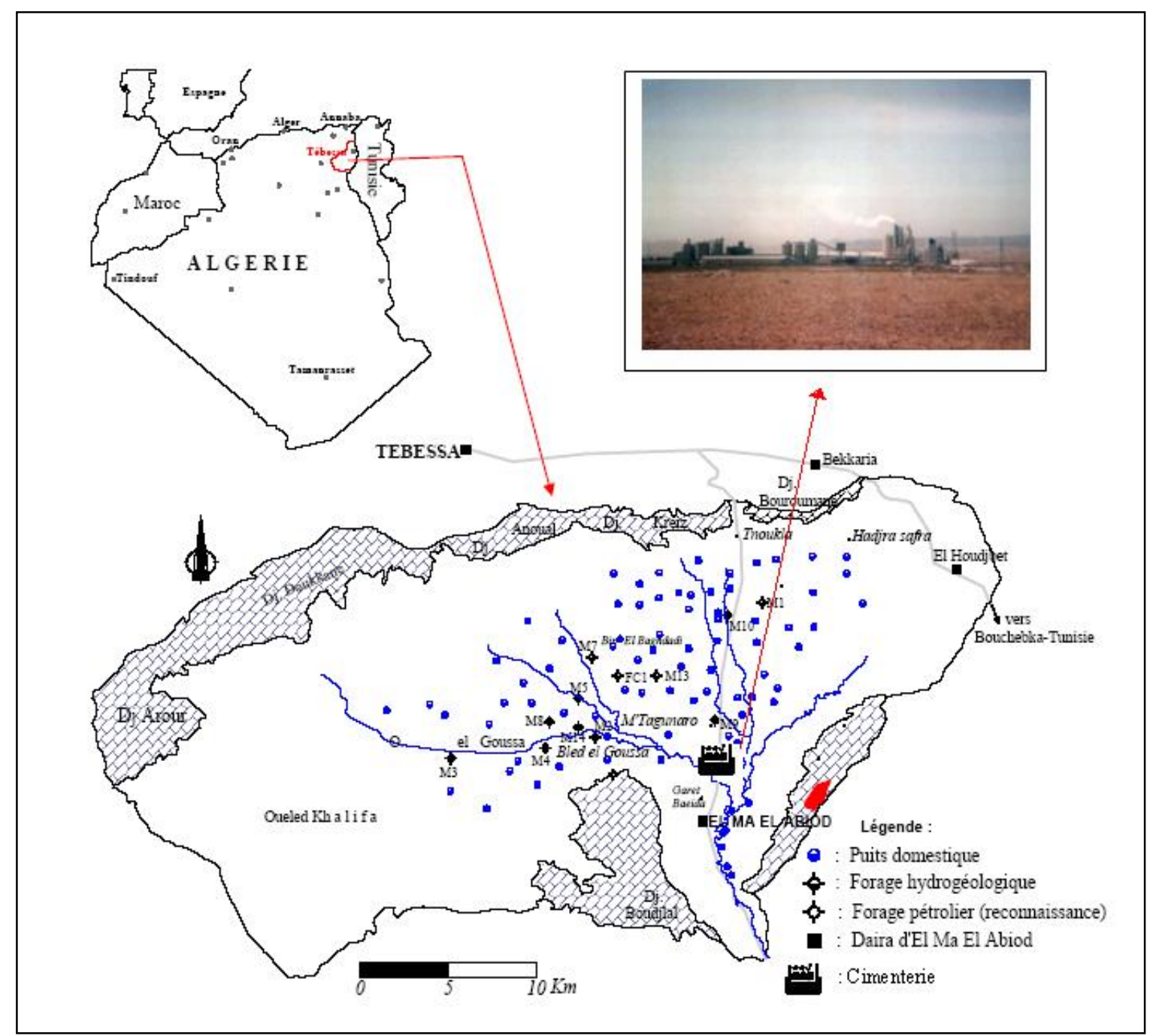

Fig.1. Situation géographique de la plaine et la cimenterie [01]

\section{Méthodologie et condition analytique}

Cette étude a pour objectif de préciser les valeurs de 7 paramètres hématologiques ; (globules rouges, hémoglobine, hématocrite, volume globulaire moyen, La concentration corpusculaire moyenne en hémoglobine, La teneur corpusculaire moyenne en hémoglobine et le nombre des globules blancs) et deux paramètres considérées comme des marqueurs précoce de l'inflammation (vitesse de sédimentation 
et le protéine-C-réactive) les travailleurs de la cimenterie ERCE et des habitants de la région d'El $\mathrm{Ma} \mathrm{El}$ Abiod et de déterminer finalement l'impact de la pollution par les rejets atmosphériques de la cimenterie sur les paramètres mesurés.

Le prélèvement du sang a été fait au niveau de l'hôpital d'El Ma El Abiod pendant une période de six mois (Avril à Septembre 2007). Il se diffère selon le type d'analyse;

- Méthodes de prélèvement pour FNS: sur tube EDTA

- Méthodes de prélèvement pour la vitesse de sédimentation (VS): sur tube à citraté

- Méthodes de prélèvement pour CRP: Le prélèvement se fait sur tube sec

Les analyses du sang ont été faites au niveau du laboratoire interne de l'hôpital de Bakkaria.

\section{Numération des cellules sanguines}

FNS ou numération formule sanguine, est un examen biologique permettant de comptabiliser les différents éléments figurés du sang. D’une manière à caractériser quantitativement les populations érythrocytaires (numérations, hématocrites, taux d'hémoglobine et indices érythrocytaires) et leucocytaires (numérations).

\section{Marqueurs précoces de l'inflammation}

Les objectifs étaient d'évaluer les réponses inflammatoires de l'organisme par calcul de deux marqueurs de l'inflammation qui sont; la vitesse de sédimentation et la protéine-C-réactif

*Protéine-C-réactif

Le CRP est une glucoprotéine du sang, synthétisé par le foie en réponse à un anti gène. En présence d'un sérum contenant de la CRP, on observe une agglutination des particules de latex due à la réaction entre CRP et son anticorps spécifique.

*Vitesse de sédimentation

La VS est la vitesse à laquelle les GR se séparent du plasma et se déposent au fond d'un tube de Westergreen. La VS est calculé selon le principe de sédimentation sur un tube gradué dans une première heure et deuxième heure. 


\section{RÉSULTATS}

Les résultats obtenus à partir de cette étude montre une ressemblance entre les travailleurs et les habitants de la région mitoyenne la cimenterie, mais des différences significatives sont apparues entre ces deux populations et les témoins pour quelques paramètres.

Les échantillons divisés pendant l'analyse statistique en deux populations, populations exposés constituées des travailleurs et des habitants proche de la cimenterie et une population témoins. L'analyse de la variance entre les deux populations se fais en utilisant le test $\mathrm{F}$ de Fisher au seuil de signification Alpha=0.050 est résumé dans le tableau suivant:

Tableau 1. Test de Fisher

\begin{tabular}{|c||c||c|c|c|c|c|c||c||c||}
\hline \hline & GR & HT & HB & VGM & TCMH & CCMH & GB & VS1 & VS2 \\
\hline \hline Fobs & 1.083 & 1.465 & 1.451 & 1.041 & $\mathbf{1 0 . 2 8 5}$ & $\mathbf{2 . 1 7 9}$ & $\mathbf{2 . 8 7 4}$ & $\mathbf{6 . 0 6 0}$ & $\mathbf{4 . 7 6 1}$ \\
\hline \hline Fcal & 1.569 & 1.569 & 1.569 & 1.669 & 1.584 & 1.596 & 1.584 & 1.456 & 1.584 \\
\hline
\end{tabular}

Au seuil de signification Alpha $=0.050$ on peut rejeter l'hypothèse nulle d'égalité des variances. Autrement dit, l'inégalité est significative pour TCMH, CCMH, GB, VS1 etVS2

Les statistiques élémentaires précédentes considéraient les variables les unes après les autres; où chaque individu est caractérisé par 09 paramètres quantitatives et des modalités illustratives ; état sanitaire, age, sexe et l'état fonctionnel. De ce point de vue, quels sont les facteurs qui se rassemblent? Quels sont ceux qui diffèrent? Plus généralement, peut-on faire une typologie des individus mettant en évidence l'ensemble ainsi défini.

D'autre part, la description des relations entre caractères peut être utile et constituer un autre objet de l'analyse. Des variables peuvent, par exemple, jouer des rôles identiques parce qu'elles sont liées par des relations particulières.

L'analyse en composantes principales permettra de répondre à ces questions. Cette méthode regroupe le traitement de description et de comparaison simultanée des différents variables.

\section{*Analyse des variances et vecteurs propres de la matrice de corrélation}

Le tableau rassemble les valeurs propres de chacune des 09 variables qui sont en combinaisons linaires de toutes les variables de départ Tableau 2. 
Tableau 2. Valeurs propres

\begin{tabular}{|c|c|c|c|c|c|c|c|c|c|c|}
\hline & F1 & F2 & F3 & F4 & F5 & F6 & F7 & F8 & F9 & F10 \\
\hline Valeur propre & 2.910 & 2.482 & 1.846 & 1.233 & 0.791 & 0.647 & 0.072 & 0.011 & 0.006 & 0.003 \\
\hline \% variance & 29.098 & 24.821 & 18.457 & 12.332 & 7.915 & 6.467 & 0.718 & 0.105 & 0.060 & 0.028 \\
\hline \% cumulé & 29.098 & 53.918 & 72.375 & 84.708 & 92.622 & 99.089 & 99.807 & 99.912 & 99.972 & 100.000 \\
\hline
\end{tabular}

La première composante principale a une variance (valeur propre) de 2.910 et représente 29.098de la variance totale, La deuxième composante principale a une variance de 2.4820 et représente 24.821 de la variabilité des données, la troisième composante principale a une variance (valeur propre) de 1.846et représente $18.457 \mathrm{de}$ la variance totale. Les trois premiers facteurs totalisent $72.375 \%$ de l'inertie totale.

\section{*Analyses des cercles}

Nous allons limiter notre observation de projection des variables et des individus au cercle formé par les axes F1 et F2, puisque il fournit la maximum d'information $(53.92 \%)$

\section{Etudes des variables}

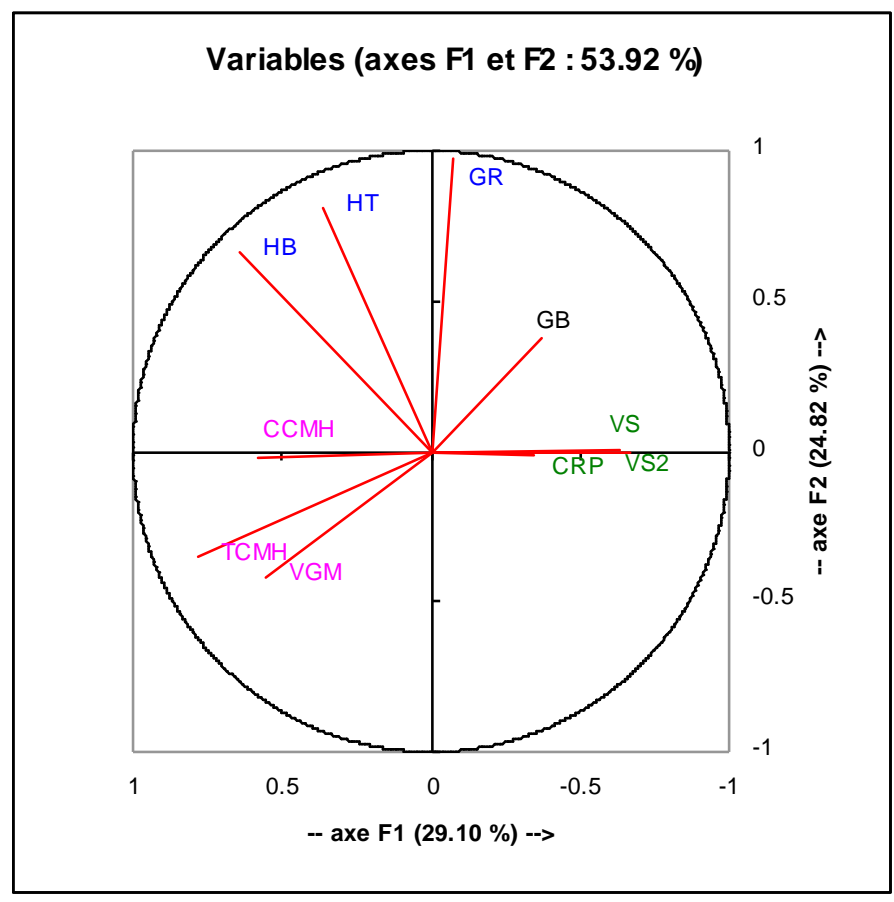

Fig.2. Représentation graphique des paramètres du sang 
L'axe F1 horizontal exprime $29.10 \%$ des informations Le nombre d'hématie, la concentration en hémoglobine et l'hématocrite sont fortement corrélés positivement au deuxième axe.

Les indices érythrocytaires, VGM, TCMH et $\mathrm{CCMH}$, sont liés positivement au premier axe.

La vitesse de sédimentation en 1 ère heure et $2^{\text {ème }}$ heure sont liées positivement au premier axe.

Les globules blancs sont opposée à tous les autres variables ce qui exprime leur nature différentes.

\section{Etudes des individus}

Le graphe suivant montre la distribution des 36 échantillons dans le plan F1 F2, il indique la ressemblance entre les échantillons et les paramètres étudiés. Figure 3

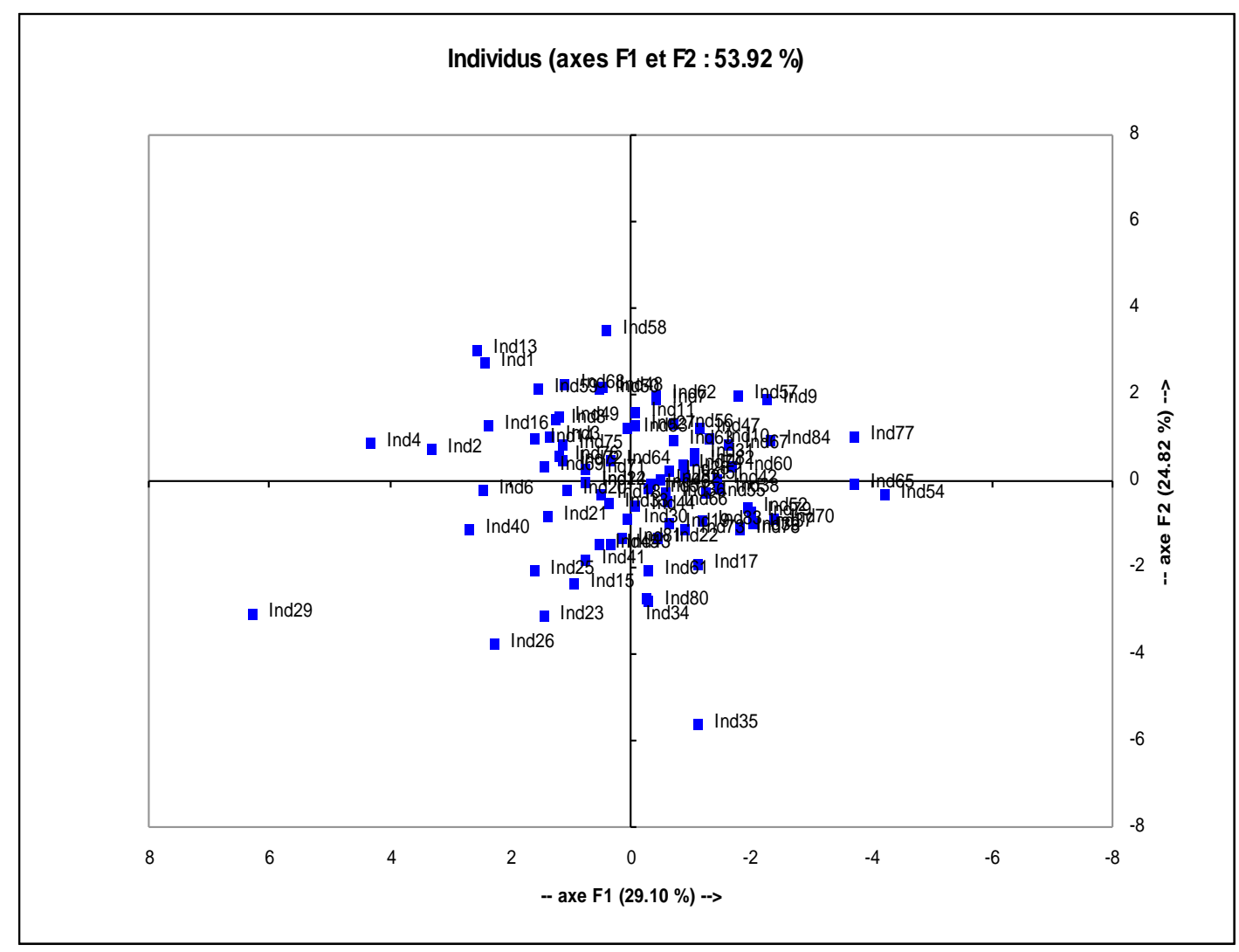

Fig.1. Projection des individus dans le plan F1 et F2 
A partir de la distribution des échantillons dans le plan factoriel F1* F2 on peut diviser les échantillons en trois groupes

Groupe 1: qui est se trouve dans la partie positive de deux axes, ce contient l'ensemble de travailleurs, ce qui explique que les conditions de travail commun entre ces individus à une influence sur les paramètres du sang. Ainsi, de point de vue numérique, les valeurs mesurées des travailleurs sont proches entre eux.

Groupe 2: qui regroupe un échantillon caractérise par 1 »attente d'une maladie respiratoire ; l'asthme, ce groupe est se rassemble dans le partie négatif des deux axe, est en comparant avec les valeurs propres de 9 variables, il apparu une homogénéité avec les valeurs de GB, VS1 etVS2 ce qui implique l'apparition des signes d'inflammation chez ces individus.

Groupe 3: ou se trouve un échantillon des prélèvements des gens sains, ce groupe est localise dans la partie positive de l'axe 1 et négative de l'axe 2.

\section{DISCUSSION}

Cette étude à permis de préciser les valeurs de 7 paramètres; hématologiques (globules rouges, hémoglobine, hématocrite, volume globulaire moyen, La concentration corpusculaire moyenne en hémoglobine, La teneur corpusculaire moyenne en hémoglobine et le nombre des globules blancs) et deux paramètres considérées comme des marqueurs précoce de l'inflammation (vitesse de sédimentation et le protéine-C-réactive) chez deux populations différents; une constituée de 84 individus infecté par la pollution de la cimenterie (travailleurs de la cimenterie et habitants) et l'autre population constituée de 70 individus considéré comme témoins.

La distribution des deux populations selon les différents outils statistiques utilisées nous permis de ressortir quelques observations ;

La comparaison de la variance des différents variables des individus exposés et témoins, montrent des différences significatives pour quelques paramètres, ainsi l'étude de l'effet de l'état sanitaire sur la variation des paramètres donne des résultats importants.

La différence significative observée pour la numération des globules blancs montre une élévation du nombre des GB chez les individus exposés est due à l'exposition des rejets atmosphériques de la cimenterie, constitué essentiellement de poussières. L'impact de la pollution atmosphérique sur le nombre des GB qui augmente dans les cas 
d'inflammation. D'autres études épidémiologiques montrent une association entre le nombre des GB et l'exposition des polluants de l'air ambiant [6].

Le nombre des GB chez la population infectée est influencé ainsi par l'état sanitaire des individus, ils sont augmentés chez les individus asmathiques qui montrent des réactions immunitaires importantes pendant les crises d'asthme.

La numération globulaire des hématies ( $\mathrm{GR}, \mathrm{Hb}, \mathrm{Ht}$, VGM, CCMH et TCMH) montre que ces paramètres ne sont pas touchées par la pollution atmosphérique a cause de leur ressemblance avec les résultats des témoins.

Des études similaires montrent que le nombre d'érythrocytes et la concentration en hémoglobine étaient négativement associés avec l'exposition aux particules fines (PM10 et PM2.5) [10], mais d'autres études expliquent la relation entre l'exposition aux polluants et l'aggravation des maladies cardio-vasculaires. L'augmentation du niveau de pollution à effet particulièrement marqué (+76\%) sur le risque global des décès de cause cardiovasculaire. L'analyse du risque relatif de décès de cause cardiovasculaire selon le niveau d'exposition aux particules fines montre une relation de type dose linéaire [8].

L'interaction entre l'état sanitaire et les paramètres GR, $\mathrm{HB}$ et $\mathrm{Ht}$ montre une association positive entre le nombre des ces derniers et l'état sanitaire ; ils sont élevés chez les asmathiques. Ce résultat est exprimé par l'activité des cellules pour la production des GR pour augmenter le nombre des molécules d'HB pour faciliter l'adhésion des molécules d' $\mathrm{O}_{2}$ ce qui facilite les phénomènes respiratoires.

Les marqueurs précoces de l'inflammation montrent des résultats importants. Les VS en 1ère et $2^{\text {ème }}$ sont élevés largement par rapport aux témoins. La présence des polluants dans l'air ambiant provoque une activité importante est essentiellement chez les gens asmathiques.

La ressemblance de cette interaction avec celle des GR montre une élévation de la viscosité du sang. Cette augmentation de la VS est l'un des signes importants de l'inflammation.

Le taux de CRP augmente dans le processus infectieux et dans la phase de nombreuses pathologies. L'absence de différence significative entre les variances des individus exposés et témoins peut liées avec les périodes de prélèvement du sang qui ne sont pas reliées directement avec les périodes de dégagement de la cimenterie ce qui erroné quelques résultats. Autrement dis, pendant les périodes de prélèvement du sang. Des variations aux niveaux de quelques paramètres mesurées n'est pas signalé c'est le cas de 
CRP. D'autres études montrent l'absence des associations positives est significative entre l'exposition récente aux particules fines et la concentration en CRP. Une autre limite et la demi-vie de la CRP, des expérimentations animales et humaines ont montré que la réponse inflammatoire après exposition aux particules prenait moins de 36 heures. Le fais d'avoir trouvés une faible association positive pour les délais plus longs [2].

L'analyse globale des de ces résultats avec l'ACP a montré une cohérence générale. L'observation des groupes a permis de détacher quelques particularités.

La ressemblance entre les paramètres et les individus montre d'une façon générale des résultats logiques.

La répartition des 9 paramètres dans le plan factoriel F1 et F2 regroupe ces paramètres en 4 groupes ;

1. Les globules blancs sont opposés par rapport aux autres paramètres à cause de leur fonction particulière dans la défense immunitaire.

2. Les GR, HB et Ht sont corrélées entre eux et fortement liées à l'axe 1. Etant aussi liées à un même variable, elles sont liées entre eux, ce qui constitue un résultat logique.

3. Les trois indices érythrocytaires VGM, CCMH et TCMH sont liées au premier axe et présente une forte relation entre eux a cause de leur origine identique.

4. Les 3 indices précoces de l'inflammation qui sont corrélés entre fortement entre eux et avec le premier axe.

Le graphe de la répartition des individus regroupes les individus en trois groupes ;

- Les travailleurs sont liés au deuxième axe

- Les individus sains qui comportent les échantillons

- Les individus asmathiques

Cette répartition montre l'effet de l'état fonctionnel et sanitaire des individus sur la variation des paramètres mesurés.

\section{REMERCIEMENTS}

Les auteurs remercient les travailleurs de la cimenterie et les personnels de l'hopital de Bakarria wilaya de Tébessa pour toutes aides. 


\section{RÉFÉRENCES}

[1] Rouabhia A. (2006). Vulnérabilité et risque de pollution des eaux souterraines de la nappe des sables miocènes de la plaine d'El Ma El Abiod Nord-Est Algerien, thèse de doctorat, université d'Annaba, 197p

[2] Aouimeur M. (2008). Impacte de la poussière du ciment sur quelques paramètres hématologiques et respiratoire cas de la cimenterie d'El Ma El Abiod, mémoire de Magister en biologie appliquée, université de Tébessa, 116p

[3] Diez Ronx A. V., Auchincloss A. H., Astor B., Barr R. G., Cushman M., Duonch T., Jacob D. R. Jr., Kautman S., Lin X., Samson P., 2006 : Exposition récente à la pollution particulaire et concentration en protéine $\mathrm{C}$ réactive (CRP) dans l'étude multiethnique sue l'anthéroséclérose, Analyse commentée par Maina L'asou et François Mathé. Revue Pollution atmosphérique. septembre 2007, 194, 3.

[4] Marieb E. N. 1993, Anatomie et physiologie humaine, 2ème édition Edition de renouveau pédagogique, Deboeck, Canada, 1014p

[5] Martin J., Maystre L. Y. 1988, Santé et pollution atmosphérique, 1er édition, ISBN, Presses polytechniques romandes, Lausanne, Suisse, $250 \mathrm{p}$

[6] Lambrozo J., Guillosso G. 2007 Pollution atmosphérique. Revue. Environnement. Risque et santé. 2008, 07(2), 99-100.

[7] Liao D., Heiss G., Chinchilmi V. M., Duan Y., Folsson A. R., Lin H. M. 2005 : Association entre les polluants atmosphériques et les taux sanguins d'indicateurs hémostatiques et d'inflammations en population urbaine. Analyses commentées par Cecile Honoré et Vincent Nedellec. Revue Pollution atmosphérique. septembre 2007, $194,3$.

[8] Poddu M., bayngana K., Tafforeau J. 2002. L'asthme et la pollution de l'air, Etat de connaissance et données disponibles pour le développement d'une politique de santé en Belgique.

[9] Miler S. Exposition prolongée à la pollution de l'air et incidence des évènements cardiovasculaires chez la femme. revue Environnement. Risques et santé. 2007, $06(04), 2$.

[10] Arqués P. 1998. La pollution de l'air, causes, conséquences, solutions, édition Edisud-ca calade, 207p. 
[11] Pernot P., Lefrance A. Pollution atmosphérique particulaire repolarisation ventriculaire. inflammation et coagulation l'étude d'Erfurt (Allemagne). revue pollution atmosphérique. 2007, 194, 6.

[12] Caquet R. 2004. 250 examens de laboratoire prescription et interprétation, 9ème édition, Masson, Paris, 453p

[13] R. Ginocchio. 1980. Energie électrique et environnement ? éditions Eyroles, Paris, $683 p$. 


\section{ETUDES DE LA VARIATION DE QUELQUES PARAMETRES SANGUINES CHEZ DES TRAVAILLEURS DE LA CIMENTERIE D 'EL MA EL ABIOD TEBESSA ALGERIE}

\section{RESUME}

En Algérie, le développement industriel engendre l'apparition des problèmes sanitaire et environnemental résultant de la pollution due à ces certaines industries tels que l'industrie cimentière. Dans le but d'évaluer l'état sanitaire des travailleurs de la cimenterie ; une étude a été faite pour 30 travailleurs de la cimenterie. Cette étude est basée sur la détermination des valeurs de 07 paramètres hématologiques (globules rouges, hémoglobine, hématocrite, volume globulaire moyen, La concentration corpusculaire moyenne en hémoglobine, La teneur corpusculaire moyenne en hémoglobine et le nombre des globules blancs) et deux paramètres considérées comme des marqueurs précoce de l'inflammation (vitesse de sédimentation et le protéine-Créactive). Ces résultats sont comparés aux deux autres populations, une des habitants de la région et l'autre des habitants loin de la cimenterie avec60 km. Les analyses statistiques sont basées sur la comparaison des variances et l'analyse en composante principale (ACP). La comparaison de la variance des différents variables des individus montre une rasemblance entre les travailleurs et les habitants proches de la cimenterie mais des différences significatives sont apparues entre les deux populations et celles de la cimenterie pour quelques paramètres. L'analyse globale des de ces résultats avec l'ACP a montré une cohérence générale. L'observation des groupes a permis de détacher quelques particularités. Cette répartition montre la relation entre l'état fonctionnel et la variation des paramètres.

Mots clés : industrie cimentière, travailleurs, signes d'inflammation, comparaison de la variance, analyse en composante principale.

How to cite this article

Aouimeur M, Goudjil T, Rouabhia A, Hamiri M. Studies of changes of some blood parameters among workers of cement of El Ma El Abiod Tebessa ALGERIA. J Fundam Appl Sci. 2009, 1(2), 58-71. 